\title{
Sindrome de allgrove en niños. Reporte de 11 casos
}

\author{
María Carmen Álvarez López, ${ }^{1}$ Pedro Coello Ramírez, ${ }^{2}$ Elizabeth García Rodríguez, ${ }^{2}$ \\ Mariana Ordoñez Cárdenas, ${ }^{1}$ Fátima Azereth Reynoso Zarzosa ${ }^{2}$

\footnotetext{
"Servicio de Gastroenterología y Nutrición Pediátrica, Hospital Civil de Guadalajara "Fray Antonio Alcalde".

2 Servicio de Gastroenterología y Nutrición Pediátrica, Hospital Civil "Juan I. Menchaca".
} \\ Guadalajara Jalisco, México.
}

Acta Gastroenterol Latinoam 2020;50(4):445-452

Recibido: 28/02/2020 / Aceptado: 23/10/2020 / Publicado online: 14/12/2020

\section{Resumen}

Introducción. El Sindrome de Allgrove es una enfermedad genética muy rara, que se hereda en forma autosómica recesiva. El gen responsable es el $A A A S$, que codifica la proteina ALADIN. Se presenta con mayor frecuencia en hijos de padres consanguineos. Se caracteriza por la triada clásica de acalasia, alacrimia, e insuficiencia suprarrenal por resistencia a la ACTH; se requiere la presencia de dos de tres manifestaciones para establecer el diagnóstico. Hay también una elevada frecuencia de sintomas neurológicos. Objetivo. Describir las caracteristicas clinicas, edad de presentación y evolución en 11 pacientes con Sindrome de Allgrove. Métodos. Se revisaron en forma retrospectiva 11 casos clínicos compatibles con Sindrome de Allgrove de presentación en la niñez. Resultados. La edad promedio al momento del diagnóstico fue de 5,9 años (rango 1-16 años). Hubo predominio del sexo femenino $(n=7)$. Los sintomas más comunes fueron vómitos postprandriales y alacrimia, presentes en el $100 \%$ de los casos al momento del diagnósti-

Correspondencia: María Carmen Álvarez López

Calle Tarascos 3514, Fracc. Monraz (C.P.: 44670). Guadalajara

Jalisco, México / Tel.: 33 38-13-04-40

Correo electrónico: carmen2000_mx@yahoo.com co. La insuficiencia suprarrenal no fue común, solamente se documentó en un paciente. Hubo consanguinidad entre los padres en el $62.5 \%$ de los casos. Conclusiones. El Síndrome de Allgrove es una causa infrecuente de disfagia, vómitos crónicos y retardo del crecimiento en niños. Ante cualquier caso documentado de acalasia se sugiere interrogar en forma dirigida la presencia de alacrimia y datos de insuficiencia suprarrenal tales como crisis convulsivas, hiperpigmentación de la piel y alteraciones neurológicas.

Palabras claves. Sindrome de Allgrove, acalasia, alacrimia.

\section{Allgrove Syndrome in Children. 11 Cas- es Report}

\section{Summary}

Background. Allgrove Syndrome is a very rare genetic disease, which is inherited in an autosomal recessive way. The responsible gene is the AAAS, that encodes the protein ALA$D I N$. It occurs most often in children of consanguineous parents. It is characterized by the classic triad of achalasia, alacrima, and adrenal insufficiency due to resistance to ACTH; the presence of two of the three previous manifestation events are required to establish the diagnosis. There is also a high frequency of the neurologic symptoms. Objective. Describe the clinical characteristics, age of presentation and evolution in 11 patients with Allgrove Syndrome. Methods. 11 clinical cases compatible with Allgrove Syndrome of presentation in childhood are retrospectively reviewed. Results. The average age at diagnosis was 5.9 years (range 1-16 years old). There was a predominance of the female sex $(n=7)$. The most common symptoms were postprandial vomiting and alacrima, 
present in 100\% of the cases at the time of diagnosis. Adrenal insufficiency was not common; it was only documented in one patient. There was consanguinity between parents in 62.5\% of the cases. Conclusions. Allgrove Syndrome is an uncommon cause of dysphagia, chronic vomiting and failure to grow in children. In case of any documented case of achalasia it is suggested to question in a directed way the presence of alacrima and adrenal insufficiency data such as seizures, hyperpigmentation of the skin and neurological alterations.

Key words. Allgrove syndrome, achalasia, alacrimia.

\section{Abreviaturas}

SA: Sindrome de Allgrove / Allgrove Syndrome. ACTH: Hormona Adrenocorticotropa.

\section{Introducción}

El Síndrome de Allgrove (SA) o síndrome de triple A (3A), es una enfermedad genética rara, que se hereda en forma autosómica recesiva, con la triada clásica de acalasia, alacrimia e insuficiencia suprarrenal por resistencia a la ACTH; algunas veces se acompaña de disfunción autonómica y alteraciones neurológicas, incluyendo retraso mental, sordera, neuropatía periférica y ataxia cerebelosa (por lo que se ha propuesto se llame síndrome 4A). ${ }^{1}$

Fue descrito en 1978 por Allgrove $^{2}$ y se debe a una mutación en el gen AAAS localizado en el cromosoma $12 q 13$, consiste en 16 exones que codifican la proteína ALADIN (alacrimia, acalasia, insuficiencia suprarrenal y trastorno neurológico). ${ }^{3}$ Es miembro de la familia de nucleoproteínas que forman el complejo poro nuclear (siglas en inglés NPC: nuclear pore complex). ALADIN está localizada en el sitio central del citoplasma del NPC, y la presencia de una mutación afecta el intercambio de material nuclear, siendo por lo tanto necesaria para la adecuada función del mismo. ${ }^{4}$ Se han identificado hasta ahora más de 70 mutaciones homocigotas o de compuestos heterocigotos, dispersos por todo el gen, excepto en el exón $3 .^{5}$

El síndrome se expresa en la primera década de la vida, diagnosticándose generalmente en la edad escolar, aunque también se ha descrito durante la tercera o cuarta década de la vida. ${ }^{6}$ Predominaen hijos de padres consanguíneos. Los síntomas de presentación son disfagia, vómitos, regurgitaciones y falta de crecimiento. ${ }^{7}$ Aunque la alacrimia no es el síntoma de presentación usual, probablemente sea el hallazgo más consistente y temprano.

Es considerada una enfermedad multisistémica, progresiva y debilitante, que puede afectar la calidad de vida de los pacientes y parece tener un curso clínico más grave comparado con los niños con acalasia idiopática. ${ }^{8}$ Los síntomas neurológicos, de los cuales las manifestaciones autonómicas son las más comunes, aparecen tardíamente. ${ }^{9}$

La alacrimia se diagnostica por la prueba de Schirmer. Esta prueba proporciona una medición semicuantitativa de la producción de lágrimas, consiste en colocar una tira reactiva estandarizada en el saco conjuntival durante un intervalo de 5 minutos; si la humedad de la tira es menor a $10 \mathrm{~mm}$ se define como alacrimia. ${ }^{9}$ La acalasia y la insuficiencia suprarrenal se diagnostican por manometría esofágica y niveles de cortisol estimulados con ACTH, respectivamente.

El presente estudio retrospectivo tiene el objetivo de describir 11 casos de SA en niños que acudieron al Servicio de Gastroenterología y Nutrición Pediátrica de los Hospitales Civiles de Guadalajara "Fray Antonio Alcalde" y Hospital Civil "Juan I. Menchaca” en un periodo de 23 años (1994-2017).

\section{Casos clínicos}

Se describen 11 pacientes, con una edad promedio al momento del diagnóstico de 5,9 años (rango 1-16 años). Hubo predominio en el sexo femenino $(\mathrm{n}=7 ; 1,7: 1)$.

Los tres primeros pacientes detectados fueron hermanos, y se consideraron compatibles con acalasia familiar sindrómica (acalasia, alacrimia y más de 2 miembros de la familia afectados). Todos los pacientes resultaron ser originarios del estado de Jalisco, siete de ellos (63,6\%) de la región de los Altos, caracterizada por una elevada consanguinidad y ancestros europeos principalmente de origen francés, portugués, italiano y flamenco, ${ }^{10}$ los restantes casos provenían del área metropolitana de la ciudad de Guadalajara.Se documentó consanguinidad entre los padres en el $62,5 \%$ de los casos; 2 de los pacientes fueron primos segundos (Tabla 1). Al interrogatorio dirigido, la madre y el abuelo materno de una paciente refirieron tener alacrimia, aunque negaron síntomas sugestivos de acalasia o de insuficiencia suprarrenal.

Los síntomas más comunes fueron vómitos postprandiales y alacrimia, se presentaron en el 100\% de los casos al momento del diagnóstico; el síntoma inicial fue el vómito, observándose desde los primeros días de vida en casi todos los pacientes (90\%). La alacrimia estuvo presente desde los primeros meses de la vida en solo 2 pacientes (18\%).

Otros síntomas reportados fueron: disfagia a sólidos y líquidos, tos crónica, infecciones recurrentes de vías respiratorias, pobre ganancia de peso, dolor torácico, sensación de asfixia, hubo además hallazgos como escotomas, pectus excavatum, lordosis y hernia inguinal bilateral (Figura 1). 
Tabla 1. Características Clínicas

\begin{tabular}{|c|c|c|c|c|c|c|c|}
\hline Paciente & $\begin{array}{l}\text { Sexo/edad } \\
\text { (años) }\end{array}$ & Síntomas iniciales & Alacrimia & Acalasia & $\begin{array}{l}\text { Disfunción } \\
\text { neurológica }\end{array}$ & Cortisol/ACTH & Consanguinidad \\
\hline 1 & $F / 4$ & Vómitos, disfagia, tos & + & + & - & Normal/Elevada & + \\
\hline 2 & $\mathrm{~F} / 6$ & Vómitos, falla para crecer, disfagia & + & + & - & Normal/Normal & + \\
\hline 3 & $\mathrm{~F} / 5$ & Vómitos, disfagia & + & + & - & Normal/Normal & + \\
\hline & & & & & & $? / ?$ & \\
\hline 4 & $F / 16$ & Vómitos, disfagia & + & + & $?$ & $\begin{array}{l}\text { convulsiones } \\
\text { presentes }\end{array}$ & + \\
\hline 5 & $M / 4$ & Vómitos, disfagia, tos & + & + & + & Normal/Elevada & + \\
\hline 6 & $\mathrm{M} / 1$ & Vómitos, falla para crecer & + & + & - & Normal/Normal & + \\
\hline 7 & $M / 13$ & Vómitos, disfagia, falla para crecer & + & + & - & Normal/Normal & - \\
\hline 8 & $F / 3$ & $\begin{array}{l}\text { Vómitos, disfagia, hiperpigmentación } \\
\text { cutánea }\end{array}$ & + & + & + & Muy bajo/? & - \\
\hline $9^{*}$ & $\mathrm{~F} / 4$ & Vómitos, tos crónica & + & + & - & $? / ?$ & $?$ \\
\hline $10^{\star}$ & $\mathrm{F} / 6$ & Vómitos & + & + & - & $? / ?$ & $?$ \\
\hline $11^{*}$ & $\mathrm{M} / 12$ & Vómitos, tos crónica & + & + & - & $? / ?$ & $?$ \\
\hline
\end{tabular}

(?) Se desconoce.

(*) Hermanos

ACTH: Hormona adrenocorticotropa.

Figura 1. Sintomas de presentación predominantes

Pacientes

20

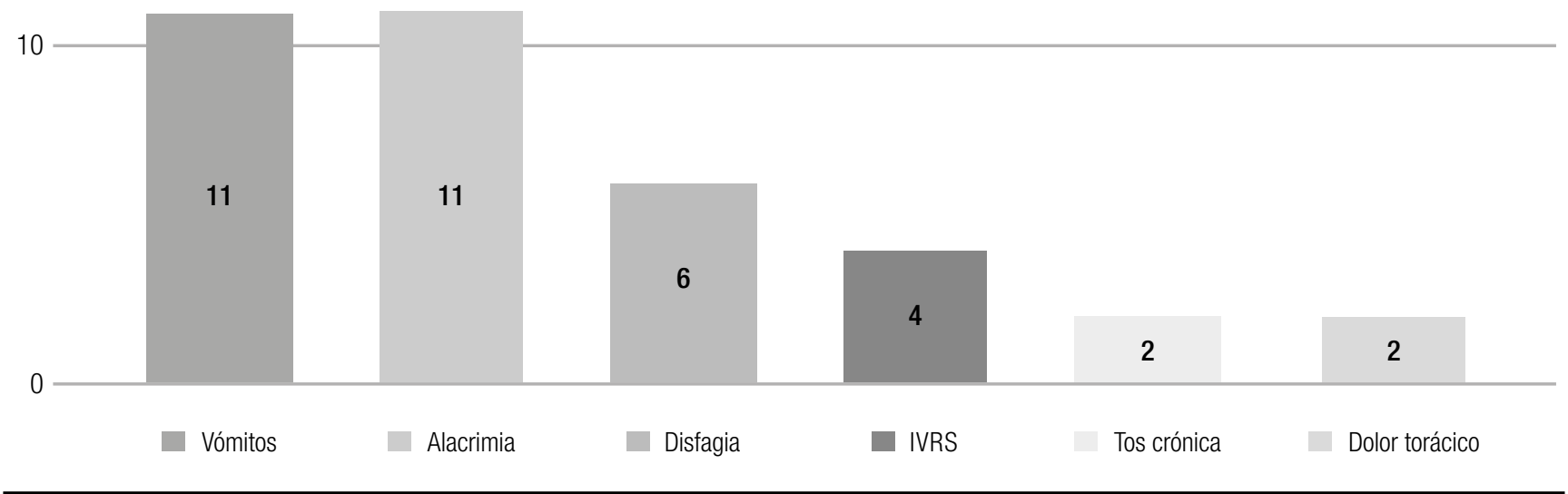


Se presentaron alteraciones neurológicas en 6 pacientes (54\%) (Tabla 2).

Tabla 2. Alteraciones neurológicas asociadas a Sindrome de Allgrove

\begin{tabular}{lc} 
Alteraciones neurológicas & Número de pacientes \\
\hline Trastornos del lenguaje & 5 \\
Retraso psicomotor & 4 \\
Trastornos del aprendizaje & 2 \\
Crisis convulsivas & 2 \\
Retraso mental & 1 \\
\hline
\end{tabular}

Los valores de cortisol y ACTH fueron normales en 10 pacientes al momento del diagnóstico, al seguimiento dos de ellos seguían teniendo valores normales de cortisol pero elevación de la ACTH. Solo un paciente presentó valores muy bajos de cortisol, hipoglucemia y crisis convulsivas a la edad de 4 años (momento del diagnóstico). No se realizó medición de cortisol después de estimulación con ACTH en ningún paciente.

El diagnóstico de acalasia se sospechó por la combinación de datos clínicos y radiológicos confirmándose mediante estudio de manometría esofágica convencional en 7 pacientes y manometría de alta resolución (MAR) en uno (Figura 2); en tres pacientes no se localizó el informe de la manometría esofágica en el expediente ignorando si contaron o no con dicho estudio. La aperistalsis esofágica se documentó en todos, en dos pacientes ocurrieron relajaciones aparentemente normales con la deglución.

Figura 2. Acalasia con compresión esofágica (subtipo II). Manometría Esofágica de Alta Resolución.

A) EEI hipertenso y sin relajaciones al momento de la deglución; $B$ ) compresión panesofágica
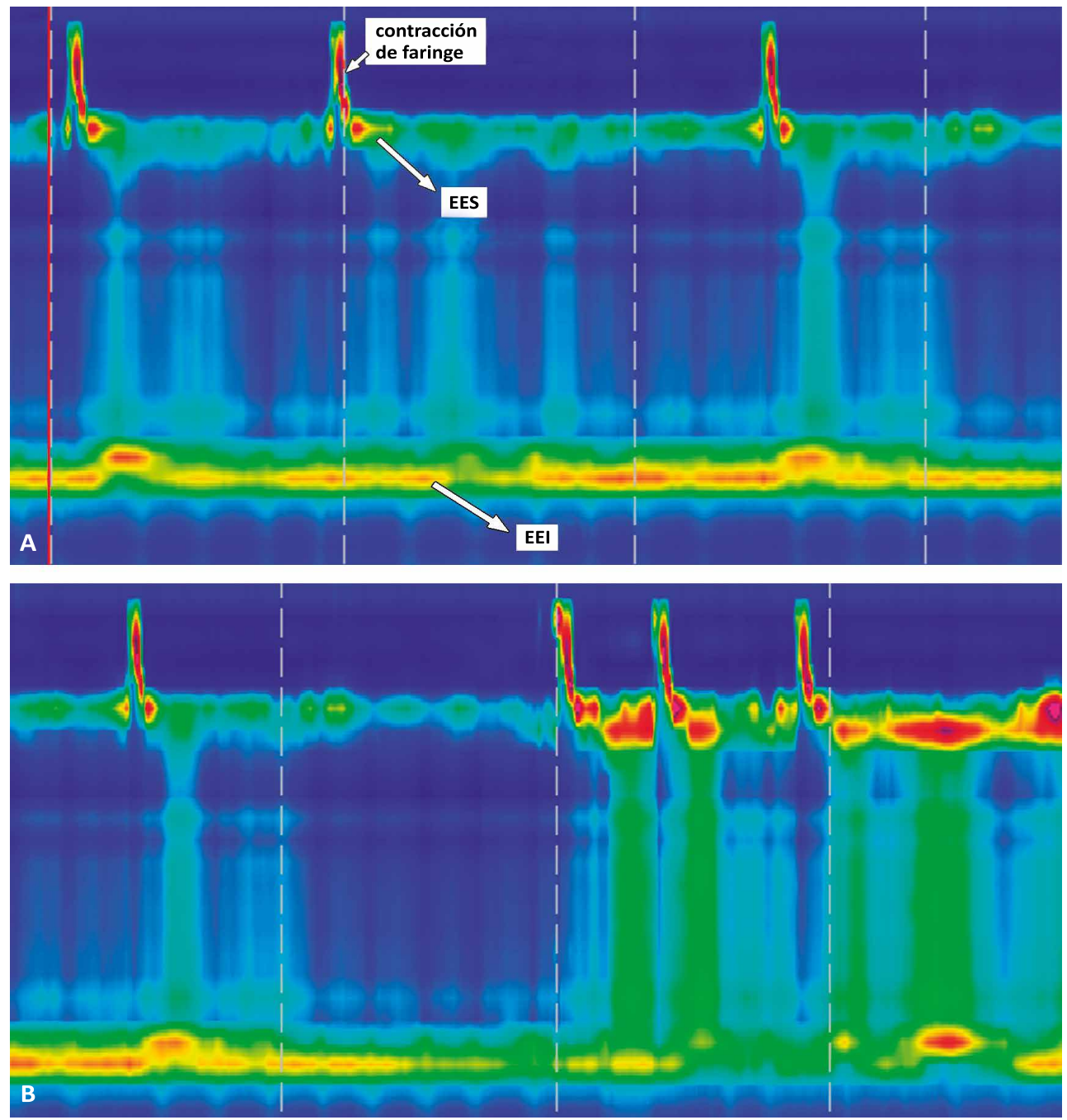

EES: Esfínter esofágico superior; EEl: Esfínter esofágico inferior. 
Los hallazgos radiológicos, manométricos y endoscópicos se muestran en la Tabla 3.
La endoscopia descartó obstrucción mecánica o pseudoacalasia en todos (Figura 3).

Tabla 3. Hallazgos endoscópicos, radiológicos y manometría esofágica

\begin{tabular}{|c|c|c|c|}
\hline $\begin{array}{l}\text { Paciente } \\
\text { sexo/edad) } \\
\text { (años) }\end{array}$ & Endoscopia & Esofagograma & Manometría esofágica \\
\hline $\mathrm{M} / 13$ & $\begin{array}{l}\text { Retención de alimentos en esófago, esofagitis } \\
\text { secundaria e infección por } C \text {. albicans. }\end{array}$ & $\begin{array}{l}\text { Megaesófago, terminación "en punta" } \\
\text { de la unión EG. }\end{array}$ & $\begin{array}{l}\text { Presión del EEl: } 70 \text { mmHg, relajación } \\
\text { ausente, aperistalsis esofágica. }\end{array}$ \\
\hline $\mathrm{F} / 4$ & Retención de alimentos en esófago. & $\begin{array}{l}\text { Megaesófago, terminación de la UEG } \\
\text { en punta. }\end{array}$ & $\begin{array}{l}\text { EEl: presión elevada } 70 \text { mmHg, rela- } \\
\text { jaciones presentes, aperistalsis eso- } \\
\text { fágica. }\end{array}$ \\
\hline $\mathrm{F} / 6$ & $\begin{array}{l}\text { Esófago dilatado, con retención de líquido } \\
\text { y alimentos. }\end{array}$ & Megaesófago. & - \\
\hline $\mathrm{F} / 5$ & $\begin{array}{l}\text { Dilatación esofágica con retención de líquido } \\
\text { y alimentos. }\end{array}$ & $\begin{array}{l}\text { Megaesófago, terminación en punta } \\
\text { de la UEG. }\end{array}$ & - \\
\hline $\mathrm{F} / 16$ & $\begin{array}{l}\text { Dilatación esofágica con retención de alimen- } \\
\text { tos, UEG cerrada. }\end{array}$ & Megaesófago, terminación en punta. & - \\
\hline$M / 4$ & $\begin{array}{l}\text { Dilatación esofágica con restos de alimentos, } \\
\text { UEG cerrada. }\end{array}$ & $\begin{array}{l}\text { Megaesófago, terminación en punta } \\
\text { de la UEG. }\end{array}$ & $\begin{array}{l}\text { EEl presión } 28 \text { mmHg, relajaciones } \\
\text { presentes, Diagnóstico de espasmo } \\
\text { esofágico difuso, un año después } \\
\text { aperistalsis franca. }\end{array}$ \\
\hline$M / 1$ & $\begin{array}{l}\text { Dilatación esofágica, con retención de líquido } \\
\text { y alimentos. }\end{array}$ & $\begin{array}{l}\text { Megaesófago, aperistalsis, terminación } \\
\text { en "pico de pájaro". }\end{array}$ & $\begin{array}{l}\text { Presión del EEl 38,6 mmHg, ausencia } \\
\text { de relajaciones, aperistalsis esofágica. }\end{array}$ \\
\hline $\mathrm{F} / 3$ & Dilatación esofágica, obstrucción de la UEG. & $\begin{array}{l}\text { Megaesófago, aperistalsis, terminación } \\
\text { en punta de la UEG. }\end{array}$ & $\begin{array}{l}\text { Presión del EEI } 90 \text { mmHg, no relaja- } \\
\text { ción, aperistalsis esofágica con presu- } \\
\text { rización panesofágica. }\end{array}$ \\
\hline
\end{tabular}

F: Femenino; M: Masculino; UEG: Unión Esofagogástrica.

EEI: Esfínter esofágico inferior.

Figura 3. Hallazgos endoscópicos y radiológicos
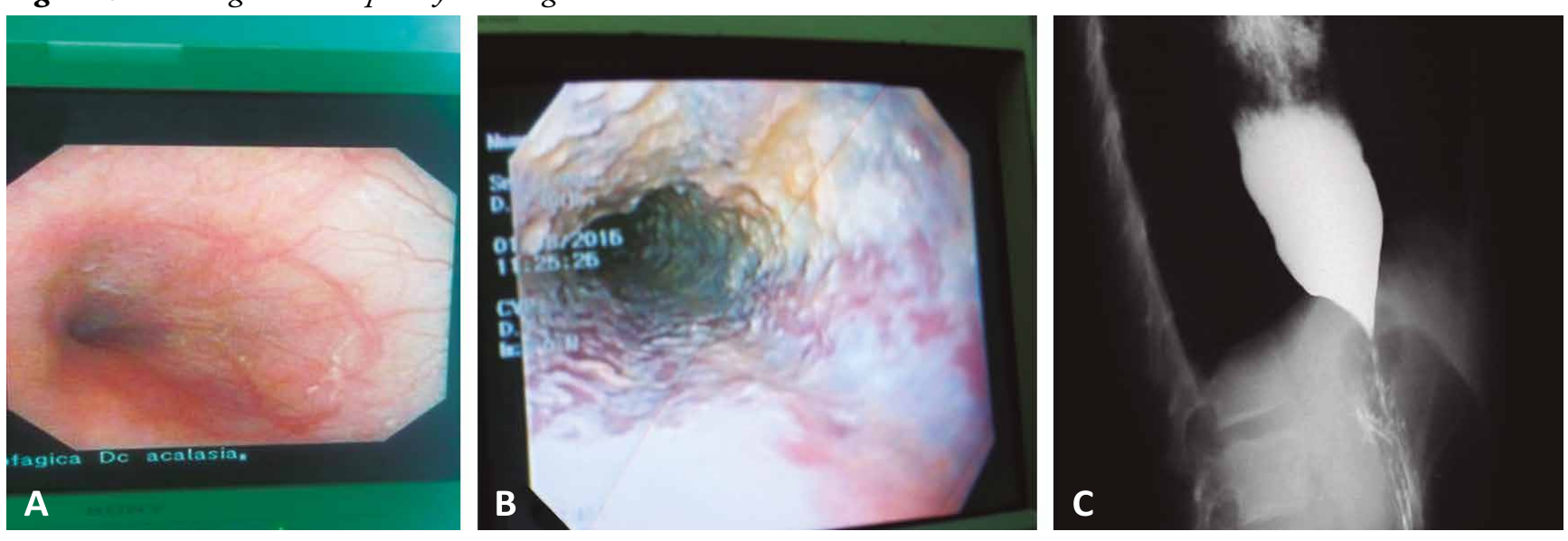

A. Unión esofagogástrica con dificultad a la apertura.

B. Retención de alimentos en el esófago e infiltrados blanquecinos compatibles con infección por C. Albicans.

C. Esofagograma que muestra dilatación esofágica y obstrucción de la unión esofagogástrica, con terminación en "punta de lápiz". 


\section{Evolución y seguimiento}

Cinco pacientes se perdieron, en los cinco el seguimiento fue de 1-3 años, y otro paciente se siguió por 16 años hasta la edad adulta. Los seis pacientes fueron sometidos a cirugía laparoscópica y se les realizó esofagomiotomía de Heller más funduplicatura. Hubo buena respuesta al tratamiento quirúrgico en los cinco pacientes, instalándose gradualmente una alimentación normal, y con ganancia de peso en todos. Un paciente persistió con disfagia importante y se operó nuevamente, ampliándose la esofagomiotomía con lo cual hubo mejoría, sin embargo, presentó síntomas de enfermedad por reflujo gastroesofágico (ERGE) y tuvo varias reintervenciones por complicaciones post-quirúrgicas (bridas, ERGE, hernia hiatal-paraesofágica). Cabe mencionar que todos los pacientes ocasionalmente presentaron disfagia sobre todo a carne, sin que esto fuese visto como un problema por el paciente o sus familiares. Todos ellos consideraron tener una evolución post-operatoria satisfactoria (Tabla 4).

Tabla 4. Tratamiento y evolución de los pacientes

\begin{tabular}{lll}
\hline $\begin{array}{l}\text { Paciente } \\
\text { sexo/edad) } \\
\text { (años) }\end{array}$ & Tratamiento & Evolución \\
\hline M/13 & Esofagomiotomía laparoscópica, y funduplicatura tipo Dor. & Mejoría, disfagia a sólidos ocasional. \\
F/4 & Esofagomiotomía laparoscópica y funduplicaturaToupet. & $\begin{array}{l}\text { Mejoría parcial, requirió reoperación por síntomas persistentes y } \\
\text { dilataciones. Complicaciones: Hernia paraesofágica, DAC, reflujo, } \\
\text { disfagia ocasional. }\end{array}$ \\
F/5 & Esofagomiotomía laparoscópica y funduplicatura. & $\begin{array}{l}\text { Mejoría parcial, ha requerido dilataciones hidroneumáticas por } \\
\text { persistencia de disfagia. }\end{array}$ \\
M/16 & Esofagomiotomía laparoscópica y funduplicaturaToupet. & Mejoría parcial, persisten vómitos y disfagia. \\
M/1 & Esofagomiotomía laparoscópica con funduplicatura. & Mejoría significativa, vómitos y disfagia ocasional. \\
\hline
\end{tabular}

M: Masculino; F: Femenino; DAC: : Dolor abdominal crónico.

\section{Discusión}

La acalasia en niños es infrecuente comparada con adultos, representa solamente el $4 \%$ a $5 \%$ de todos los casos de acalasia reportados a nivel mundial y el $10 \%$ (129 niños) informado en una serie de 1256 casos de acalasia en un estudio prospectivo realizado en Algeria. ${ }^{11} \mathrm{La}$ incidencia de SA se desconoce y en la literatura solamente se encuentran informes de casos.

En la serie de casos informada por Tebaibia y col. ${ }^{11}$ se encontró que 97/1256 (7,7\%) pacientes con acalasia tuvieron SA predominando en el sexo femenino (53\%), con una edad media de 16,23 $\pm 10,4$ años (rango 8 meses - 41 años). Se consideró SA $3 A$ en $46,4 \%(45 / 97)$, SA $2 A$ en $25,7 \%$ (25/97) y SA $4 A$ en $27,8 \%$ (27/97). Hubo consanguinidad en el 63\% (61/97), insuficiencia suprarrenal en $40 \%$ y anormalidades neurológicas en 52,6\% (51/97).

En México se desconoce la epidemiología de la aca- lasia y de SA, solo existen informes aislados de casos. En 1998 García-Compeán y col. ${ }^{12}$ describieron a un paciente de 12 años con disfagia, en quien se sospechó acalasia y se confirmó por endoscopia, esofagograma y manometría, cursando además con alacrimia y datos de disfunción neurológica y de neuropatía autonómica, con retraso psicomotor y bajo coeficiente intelectual. La prueba de estimulación suprarrenal con ACTH resultó normal. Martínez-Guzmán y col. ${ }^{13}$ describieron un paciente de 7 años con alacrimia, atrofia óptica y antecedentes de acalasia sin insuficiencia suprarrenal. González-Rodríguez y col. ${ }^{14}$ informaron un paciente de 17 años con diagnóstico de alacrimia a los 18 meses de edad quien cursó con úlceras corneales, zonas hiperpigmentadas en pliegues e insuficiencia suprarrenal y neuropatía motora axonal; a los 7 años presentó disfagia y se corroboró acalasia.

La acalasia infantil puede presentar varias formas: a) Acalasia clásica no familiar o esporádica (afecta a un 
solo miembro de una familia); b) Acalasia familiar (por lo menos dos miembros de una familia están afectados; c) SA cuando se encuentran dos de los tres siguientes datos: acalasia, alacrimia o insuficiencia suprarrenal; y por último d) acalasia familiar sindrómica (dos o más miembros de una familia tienen acalasia y datos de SA). ${ }^{11}$

En el informe de Tebaibia y col. de 1256 pacientes con acalasia, 129 (10\%) fueron niños, con una prevalencia muy alta de acalasia familiar sindrómica, observándose en el 45\% (58/129), acalasia clásica no familiar o esporádica en el 55\% (71/129). La alta prevalencia de SA está relacionada probablemente con la tradición de matrimonios consanguíneos en Algeria, al igual que en la serie aquí reportada.

Nuestros pacientes presentaron síntomas desde el primer año de vida; el vómito fue el síntoma principal; estuvo presente desde el nacimiento en el $90 \%$ de los casos, confundiéndose con ERGE, originando que el diagnóstico se retrasara hasta una edad promedio de 5,9 años o hasta la adolescencia. La insuficiencia suprarrenal no fue común y solo un paciente la presentó al momento del diagnóstico con deficiencia de cortisol, hipoglucemia y convulsiones. Al seguimiento, dos pacientes más tuvieron elevación discreta de ACTH y cortisol normal. Estos resultados contrastan con lo descrito en la literatura, ya que después de la alacrimia, la insuficiencia suprarrenal es el síntoma más común. ${ }^{8}$

De acuerdo con la clasificación de acalasia referida por Tebaibia y col., ${ }^{11}$ ocho de nuestros pacientes (72\%) corresponden a un SA 2A caracterizado por alacrimia y acalasia, 1 fue compatible con SA 3A (alacrimia, acalasia e insuficiencia suprarrenal); tres miembros de una sola familia presentaron acalasia y alacrimia, por lo que se consideraron como SA familiar o acalasia familiar sindrómica (27\%); 6/11 (54\%) tuvieron alteraciones neurológicas importantes, con un porcentaje muy similar al descrito por dichos autores. ${ }^{11}$

La alacrimia presente en todos los pacientes al momento del diagnóstico, solo fue observado tempranamente por los familiares en dos de ellos (18\%) a diferencia de lo informado en la literatura. ${ }^{15}$

La insuficiencia suprarrenal no ocurre inmediatamente después del nacimiento, sino que resulta de un proceso progresivo que conduce a hipofunción de las suprarrenales en un tiempo variable, pudiendo preservarse niveles normales de cortisol hasta la tercera década de la vida. ${ }^{16}$ En una revisión de 20 casos la deficiencia de cortisol estuvo presente en todos, con un inicio de síntomas entre 1-8, 3 años. ${ }^{9}$ La razón exacta para la insuficiencia suprarrenal no es clara, puede ocurrir como resultado de un proceso progresivo que conduce a hipofunción de la glándula en un tiempo variable después del nacimiento. ${ }^{16} \mathrm{La}$ actividad de los mineralocorticoides parece estar respetada en la gran mayoría de los casos informados.

La insuficiencia suprarrenal fue poco común en nuestra serie, aunque es probable que no haya sido detectada al no realizarse la prueba de estimulación con ACTH.

$\mathrm{La}$ asociación frecuente con alteraciones neurológicas, dermatológicas, dismorfias faciales, disautonomía, talla baja y osteoporosis hacen que esta entidad sea considerada una enfermedad multisistémica e incapacitante, lo cual ensombrece el pronóstico y la calidad de vida de los pacientes.

La manometría esofágica es el estándar de oro para el diagnóstico de acalasia. En centros donde no se cuenta con este recurso, el diagnóstico se realiza en base a los síntomas clínicos, hallazgos endoscópicos y radiológicos. Las nuevas técnicas de manometría esofágica de alta resolución permiten una mejor definición del perfil manométrico de la acalasia. ${ }^{17,}{ }^{18} \mathrm{La}$ aperistalsis esofágica es el hallazgo manométrico característico en los pacientes con acalasia, también puede observarse hipertensión y falta de relajación del esfínter esofágico inferior. Sin embargo, la función del esfínter esofágico inferior puede ser heterógenea encontrando relajaciones aparentemente normales después de la deglución en algunos pacientes, tal como fue informado por Morera y Nurko; ${ }^{19}$ este hallazgo se pudo observar en dos de nuestros pacientes (Tabla 3).

La acalasia infantil debería conducirnos a buscar otros casos similares en la familia con el fin de detectar casos asintomáticos de SA. La alacrimia y la consanguinidad entre los padres deben identificarse mediante interrogatorio dirigido.

El estudio genético es muy importante para la clasificación de los casos de acalasia que incluyen SA y deberá realizarse cuando exista disponibilidad.

Aunque no es el objetivo de este trabajo, se informa la evolución y respuesta al tratamiento quirúrgico en 6 pacientes (se perdió el seguimiento en los restantes) (Tabla 4). Cabe mencionar que debido al carácter retrospectivo del trabajo y el seguimiento errático no pueden sacarse conclusiones acerca de la efectividad del tratamiento.

Es relevante el número de casos de SA encontrados en nuestro centro hospitalario, comparado con los nueve pacientes descritos por Alhussaini y col. que provenían de tres centros de Gastroenterología Pediátrica en Francia y Bélgica. ${ }^{8}$

La importancia de esta revisión es alertar a la comunidad médica sobre la existencia de esta condición . Reiteramos la necesidad del interrogatorio dirigido a las familias donde exista un caso de acalasia con el fin de encontrar casos no diagnosticados. 


\section{Sostén financiero. No se recibió ninguno.}

\section{Referencias}

1. Gazarian C, Cowell CT, Bonney M, Grigor W. The '4A' syndrome: Adrenocortical insufficiency associated with achalasia, alacrima, autonomic and other neurological abnormalities. Eur J Pediatr 1995; 154: 18-23.

2. Allgrove J, Clayden GS, Grant DB. Familial glucocorticoid deficiency with achalasia of the cardia and deficient tear production. Lancet 1978; 1: 1284-1286.

3. Papageorgiou L, Mimidis K, Katsani KR, Fakin G. The genetic basis of triple A (Allgrove) syndrome in a Greek family. Gene 2013; 512: 505-509.

4. Kind B, Koehler K, Lorenz M, Huebner A. The nuclear pore complex protein ALADIN is anchored via NDC1 but not via POM121 and GP210 in the nuclear envelope. Biochem Biophys Res Commun 2009; 390: 205-210.

5. Huebner A, Kaindl AM, Knobeloch KP, Petzold H, Mann P, and Koehler $\mathrm{K}$. The triple A syndrome is due to mutations in ALADIN, a novel member of the nuclear pore complex. Endocr Res 2004; 30: 891-899.

6. Pedreira CC, Zacharin MR. Allgrove syndrome: When a recognizable paediatric disorder occurs in adulthood. MJA 2004; 180: 74-75.

7. Brooks BP, Kleta R, Stuart C, Tuchman M, Jeong A, Stergiopoulus SG, Bei T, Bjornson B, Russell L, Chanoine J-P, Tsagarakis S, Kalsner LR, Stratakis CA. Genotypic heterogeneity and clinical phenotype in triple A syndrome: a review of the NIH experience 2000-2005. Clin Genet 2005; 68: 215-221.

8. Alhussaini B, Gottrand F, Goutet J-M, Scaillon M, Michaud L, Spyckerelle $\mathrm{C}$, Viola S, Lamblin $\mathrm{M}-\mathrm{D}$. Clinical and manometric characteristics of Allgrove syndrome. JPGN 2011; 53: 271-274.

9. Grant DB, Barnes ND, Dumic M, Ginalska-Malinowska M, Milla PJ, Ptrykowski Wv, Rowlatt RJ, Steendijk R, Wales JHK, Werder E. Neurological and adrenal dysfunction in the adrenal insufficiency/alacrima/ achalasia (3A) syndrome. Arch Dis Child 1993; 68: 779-788.
10. Disponible en: htpp://www.fazendogenero.ufsc.br/8sts/ST65/Ortiz-Cortes 65.pdf

11. Tebaibia A, Amine M, Boutarene D, Benmediouni F, Oumnia N. Incidence, clinical features and para-clinical findings of achalasia in Algeria: Experience of 25 years. World J Gastroenterol 2016; 22: 8615-8623.

12. Garcia-Compeán D, Martínez HR, Villegas-González MJ, Montes J, García F, González JA. Acalasia, alacrimia sin insuficiencia suprarrenal, con disfunción neurológica periférica y autonómica (Sindrome de Allgrove). Rev Gastroenterol Mex 1998; 63: 33-36.

13. Martínez-Guzmán O, Hernández-Abrego MP, Villanueva-Mendoza C. Síndrome de Allgrove: Reporte de un caso, Bol Med Hosp Infant Mex 2009; 66: 76-79.

14. González R, Serrano M, Montiel AJ, Alvarado I, Barragán RE: Síndrome de Allgrove, Informe de un caso. Rev Fac Med UNAM 2016; 59: 16-19.

15. Zamanfar D, Shokri E, Shadani S, Shahmohammadi S. Allgrove's Syndrome: Two cases reports and review of literature. J Pediatr Rev 2015; 3: 48-52.

16. Lanes R, Plotnick LP, Bynum TE, Lee PA, Casella JF, Fox CE, Kowarsky AA, Migeon CJ. Glucocorticoid and partial mineralocorticoid deficiency associated with achalasia. J Clin Endocrinol Metab 1980; 50: 268-270.

17. Bredenoord A, Kharilas P, Pandolfino J, Schwizer W, Smout AJPM, Conklin J, Cook I, Gyawali P, Hebbard G, Holloway R, Ke M, Keller J, Mittal R, Petters J, Rickter J, Roman S, Rominel N, Siffrim D, Tutuian R, Valdovinos M, Vela MF, Zerbib F. Chicago Classification Criteria of Esophageal Motility Disorders Defined in High Resolution Esophageal Pressure Topography (EPT). Neurogastroenterology Motil 2012; 24 (Suppl 1): 57-65.

18. Van Lennep, van Wijk, Omari T, Salvatore S, Benninga M, Singendonk M. Clinical Management of Pediatric Achalasia: A Survey of Current Practice. JPGN 2019; 68: 521-526.

19. Morera C, Nurko S. Heterogeneity of Lower Esophageal Sphincter Function in Children with Achalasia. JPGN 2012; 54: 34-40. 